\title{
An Inertial Accelerated Algorithm for Solving Split Feasibility Problem with Multiple Output Sets
}

\author{
Huijuan Jia, ${ }^{1,2}$ Shufen Liu, ${ }^{1}$ and Yazheng Dang $\mathbb{D}^{3}$ \\ ${ }^{1}$ College of Computer Science and Technology, Jilin University, 2699 Qianjin Street, Chaoyang District, \\ Changchun 130012, China \\ ${ }^{2}$ College of Computer Science and Technology, Henan Polytechnic University, 2001 Century Avenue, \\ Shanyang District, Jiaozuo 454003, China \\ ${ }^{3}$ Department of Management, Shanghai University of Science and Technology, Shanghai PRC 200093, China
}

Correspondence should be addressed to Yazheng Dang; jgdyz@163.com

Received 4 June 2021; Accepted 6 October 2021; Published 5 November 2021

Academic Editor: Antonio Di Crescenzo

Copyright (c) 2021 Huijuan Jia et al. This is an open access article distributed under the Creative Commons Attribution License, which permits unrestricted use, distribution, and reproduction in any medium, provided the original work is properly cited.

The paper proposes an inertial accelerated algorithm for solving split feasibility problem with multiple output sets. To improve the feasibility, the algorithm involves computing of projections onto relaxed sets (half spaces) instead of computing onto the closed convex sets, and it does not require calculating matrix inverse. To accelerate the convergence, the algorithm adopts self-adaptive rules and incorporates inertial technique. The strong convergence is shown under some suitable conditions. In addition, some newly derived results are presented for solving the split feasibility problem and split feasibility problem with multiple output sets. Finally, numerical experiments illustrate that the algorithm converges more quickly than some existing algorithms. Our results extend and improve some methods in the literature.

\section{Introduction}

Let $H_{1}$ and $H_{2}$ be two real Hilbert spaces and $C$ and $Q$ be nonempty, closed, and convex subsets of $H_{1}$ and $H_{2}$, respectively. Let $B: H_{1} \longrightarrow H_{2}$ be a nonzero bounded linear operator and $B^{T}$ be its adjoint.

The split feasibility problem (SFP) is formulated to find a point $x^{*} \in \mathrm{H}_{1}$ satisfying

$$
x^{*} \in \mathrm{C} \text { such that } B x^{*} \in Q \text {. }
$$

The SFP was introduced in [1], which has broad applications in many fields such as image reconstruction problem $[2,3]$ and intensity-modulated radiation therapy (IMRT) [4-6]. Byrne [2] introduced the CQ-algorithm for solving (1) as follows:

$$
x^{0} \in H_{1} ; x^{k+1}:=P_{C}\left(x^{k}-\tau_{k} B^{T}\left(I-P_{Q}\right) B x^{k}\right),
$$

where $P_{C}$ and $P_{Q}$ are the metric projections onto $C$ and $Q$, respectively, that is, $P_{C}(x)=\operatorname{argmin}_{y \in C}\|x-y\|$, over all $x$ C. $\tau_{k} \in\left(0,2 / B^{2}\right)$, where $B^{2}$ is the spectral radius of the matrix $B^{\mathrm{T}} B$.

In 2004, Yang [7] made the following assumptions.

(1) The sets $C$ and $Q$ are denoted as

$$
C:=\left\{x \in H_{1}: c(x) \leq 0\right\} \text { and } Q=\left\{y \in H_{2}: q(y) \leq 0\right\},
$$

where $c: H_{1} \longrightarrow \mathbb{R}$ and $q: H_{2} \longrightarrow \mathbb{R}$ are convex and subdifferential functions on $H_{1}$ and $H_{2}$, respectively.

(2) The subgradients $\partial c(x)$ and $\partial q(y)$ of $c$ and $q$, respectively, can be calculated; they are 


$$
\begin{aligned}
\partial c(x):= & \left\{\xi \in H_{1}: c(z) \geq c(x)+\xi, z\right. \\
& \left.-x, \text { for each } z \in H_{1}\right\}, \\
\partial q(y):= & \left\{\eta \in H_{2}: q(z) \geq q(x)+\eta, u\right. \\
& \left.-y, \text { for each } u \in H_{2}\right\},
\end{aligned}
$$

which are bounded operators (i.e., bounded on bounded sets).

Define two half-spaces $C_{k}$ and $Q_{k}$ as

$$
C_{k}:=\left\{x \in H_{1}: c\left(x^{k}\right) \leq \xi^{k}, x^{k}-x\right\},
$$

where $\xi^{k} \in \partial c\left(x^{k}\right)$ and

$$
Q_{k}:=\left\{y \in H_{2}: q\left(B x^{k}\right) \leq \eta^{k}, B x^{k}-y\right\},
$$

where $\eta^{k} \in \partial q(B x)$. It is easy to see that $C_{k} \supset C$ and $Q_{k} \supset Q$, for all $k \geq 1$.

Yang, in [7], introduced the following relaxed CQalgorithm for solving the SFP (1) in a finite-dimensional Hilbert space:

$$
x^{0} \in H_{1} ; x^{k+1}:=P_{C_{k}}\left(x^{k}-\tau_{k} \nabla f_{k}\left(x^{k}\right)\right),
$$

where $\quad f_{k}\left(x^{k}\right):=(1 / 2)\left(I-P_{Q_{k}}\right)^{2}, \quad \nabla f_{k}\left(x^{k}\right):=B^{T}(I-$ $\left.P_{Q_{k}}\right) B x^{k}$, and $\tau_{k} \in\left(0,2 / B^{2}\right)$. Since $P_{C_{k}}$ and $P_{Q_{k}}$ are easily calculated, this method appears to be very practical. However, to compute the norm of B turns out to be complicated and costly. To overcome this difficulty, in 2012, L'opez et al. [8] introduced a relaxed CQ algorithm for solving the SFP (1) with a new adaptive way of determining the stepsize sequence $\tau_{k}$ defined as follows:

$$
\tau_{k}:=\frac{\rho_{k} f_{k}\left(x^{k}\right)}{\nabla f_{k}\left(x^{k}\right)^{2}}
$$

where $\left\{\rho_{k}\right\} \in(0,4), \forall k \geq 1$, such that inf ${ }_{n \rightarrow \infty} \rho_{k}\left(4-\rho_{k}\right)>0$. It was proved that the sequence $\left\{x^{k}\right\}$ generated by (8) with $\tau_{k}$ defined by (9) converges weakly to a solution of the SFP (1). That is, their algorithm has only weak convergence in the framework of infinite-dimensional Hilbert spaces.

Many authors also proposed algorithms that generate a sequence $\left\{x^{k}\right\}$ which converges strongly to a point in the solution set of the SFP (1), see, e.g., [9-12]. In particular, Deepho and Kumam [11] proposed a modified Halpern's iterative scheme for solving the SFP (1) in the setting of infinite-dimensional Hilbert spaces as follows:

$$
\begin{aligned}
u, x^{0} \in H_{1} ; x^{k+1}:= & \beta_{k} u+\delta_{k} x^{k} \\
& +\gamma_{k} P_{C}\left(x^{k}-\tau_{k} B^{*}\left(I-P_{Q}\right) B x^{k}\right), \forall k \geq 1,
\end{aligned}
$$

where $0<\tau_{k}<2 / B^{2}$ and $\left\{\beta_{k}\right\},\left\{\delta_{k}\right\}$, and $\left\{\gamma_{k}\right\}$ are three sequences in $[0,1]$ such that $\beta_{k}+\delta_{k}+\gamma_{k}=1$. Under some mild and standard assumptions, it was proved that the sequence $\left\{x^{k}\right\}$ generated by (10) converges strongly to a solution of the SFP (1).
Recently, Reich et al. [13] considered and studied the following split feasibility problem with multiple output sets in Hilbert spaces.

Let $H$ and $H_{i}, i=1, \ldots, N$, be real Hilbert spaces and let $B_{i}: H \longrightarrow H_{i}, i=1, \ldots, N$, be bounded linear operators. Let $C$ and $Q_{i}, i=1, \ldots, N$, be nonempty, closed, and convex subsets of $H$ and $H_{i}, i=1, \ldots, N$, respectively. Given $H, H_{\mathrm{i}}$, and $B_{\mathrm{i}}$ as above, the split feasibility problem with multiple output sets (in short, SFPwMOS) is to find an element $x^{*}$ such that

$$
x^{*} \in \Gamma:=C \cap\left(\cap{ }_{i=1}^{N} B_{i}^{-1}\left(Q_{i}\right)\right) \neq 0 .
$$

Reich et al. defined the function $g: H \longrightarrow \mathbb{R}$ by

$$
g(x):=1 / 2 \sum_{i-1}^{N}\left(I-P_{Q_{i}}\right) B_{i} x^{2}, \text { for all } x \in H .
$$

It is not difficult to see that an element $p^{*}$ is a solution to the SFPwMOS (11) if and only if it is a solution to the problem

$$
\min _{x \in C} g(x)
$$

This is equivalent to

$$
0 \in \nabla g\left(x^{*}\right)+N_{C}\left(x^{*}\right),
$$

where $N_{C}(x)$ is the normal cone of $C$ at the point $x$ (recall : let $C \subset H$ be a closed convex subset of a real Hilbert space $H$. The normal cone of $C$ at $x$ denoted by $N_{C}(x)$ is given by $\left.N_{C}(x)=\{z \in H: z, y-x \leq 0, \forall y \in C\}\right)$, which implies

$$
x^{*}=P_{C}\left(x^{\mathrm{T}}-\lambda \sum_{i=1}^{N} B_{i}^{T}\left(I-P_{Q_{i}}\right) B_{i} x^{\mathrm{T}}\right) \text {, }
$$

where $\lambda$ is an arbitrary positive real number. Motivated by these characterizations, Reich et al. [13] introduced the following iterative method for solving the SFPwMOS (11). For any given point, $x^{0} \in H,\left\{x^{k}\right\}$ is generated by the following iteration:

$$
x^{k+1}:=\alpha_{k} f\left(x^{k}\right)+\left(1-\alpha_{k}\right) P_{C}\left(x^{k}-\lambda_{k} \sum_{i=1}^{N} B_{i}^{T}\left(I-P_{Q_{i}}\right) B_{i} x^{k}\right) \text {, }
$$

where $f: C \longrightarrow C$ is a strict contraction mapping of $H$ into itself with the contraction constant $\theta \in[0,1), \lambda_{k} \subset(0, \infty)$ and $\left\{\alpha_{k}\right\} \subset(0,1)$. It was proved that if the sequences $\left\{\lambda_{k}\right\}$ and $\left\{\alpha_{k}\right\}$ satisfy the conditions,

$$
(I-f) x^{*}, x-p x^{*} \geq 0 \forall y \in \Gamma .
$$

An important observation here is that the method (Scheme (17)) introduced by Reich et al. [13] requires to compute the metric projections on to the sets $C$ and $Q_{i}$. Moreover, it needs to compute the operator norm, and it only employs current iterative point to obtain the next iteration, which leads to slow convergence.

Inertial effect was proposed by Polyak [14] to speed up convergence of smooth convex minimization problem. The 
main idea is to make use of two previous iterates in order to update the next iterate. Due to its good acceleration character, inertial type algorithms have been widely studied by authors [15-19].

Motivated by the modified Halpern's iterative scheme proposed by [11] for the SFP (1), the relaxed CQ algorithm is proposed by He et al. [11] to solve the SFP (1) and inertial effect is proposed by Polyak [14]. In this paper, we propose a new inertial self-adaptive relaxed CQ algorithm for solving the SFPwMOS (11) in general Hilbert spaces.

In Section 2, we recall some necessary tools which are used in establishing our main results. In Section 3, we propose an inertial self-adaptive relaxed CQ algorithm for solving the SFPwMOS (11), and we establish and analyze a strong convergence theorem for the proposed algorithm. In Section 4, we present some newly derived results for solving the SFPwMOS (11). Finally, in Section 5, we provide one numerical experiment to illustrate the implementation of our proposed method and compare it with some existing results.

\section{Preliminaries}

In this section, we recall some preliminaries which are needed in the sequel. Let $H$ be a real Hilbert space with the inner product $\langle.,$.$\rangle and induced norm |\cdot|$. Let $I$ stand for the identity operator on $H$. We denote the fixed point set of an operator $T: H \longrightarrow H$ (if $T$ has fixed point) by Fix $(T)$, i.e., $\operatorname{Fix}(T)=\{x \in H: T x=x\}$. Let the symbols" " and " $\longrightarrow$ " denote the weak and strong convergence, respectively. For any sequence, $\left\{x^{k}\right\} \subset H, \omega_{\omega}\left(x^{k}\right)$ $=\left\{x \in H: \exists\left\{x^{k^{j}}\right\} \subset\left\{x^{k}\right\}\right\}$ such that $\left\{x^{k^{j}} \rightarrow x^{k}\right\}$ denotes the weak $\omega$-limit set of $\left\{x^{k}\right\}$.

Definition 1 (see [3]). Let $H$ be a real Hilbert space with inner product $\langle$,$\rangle and induced norm |\cdot|$. Let $C$ be a nonempty closed convex subset of $H$. Let $T: C \longrightarrow H$ be a given operator. Then, $T$ is called

(1) Lipschitz continuous with constant $\sigma>0$ on $C$ if

$$
T x-T y \leq \sigma x-y, \forall x, y \in C .
$$

(2) Nonexpansive on $C$ if

$$
T x-T y \leq x-y, \forall x, y \in C \text {. }
$$

(3) Firmly nonexpansive on $C$ if

$$
T x-T y^{2} \leq x-y^{2}-(I-T) x-(I-T) y^{2}, \forall x, y \in C,
$$

which is equivalent to

$$
T x-T y^{2} \leq T x-T y, x-y, \forall x, y \in C .
$$

(4) Averaged if there exists a number $\sigma \in(0,1)$ and a nonexpansive operator $F: C \longrightarrow H$ such that

$$
T=F+(1-\sigma) I
$$

where $I$ is the identity operator.

In this case, we say that $T$ is $\sigma$-averaged.

Lemma 1. Let $C \subset H$ be a nonempty, closed, and convex set, and $P_{C}(x)$ denotes the metric (orthogonal) projection of $x$ onto $C$. Then, the following assertions hold for any $x, y \in H$ and $z \in C$ :

$$
\begin{aligned}
& \text { (1) } x-P_{C}(x), z-P_{C}(x) \leq 0 \\
& \text { (2) } P_{C}(x)-P_{C}(y) \leq x-y \\
& \text { (3) } P_{C}(x)-P_{C}(y)^{2} \leq P_{C}(x)-P_{C}(y), x-y, \\
& \text { (4) } P_{C}(x)-z^{2} \leq x-z^{2}-x-P_{C}(x)^{2} .
\end{aligned}
$$

Evidently, $I-P_{C}$ is firmly nonexpansive and also nonexpansive.

Definition 2. Let $\delta \in(0,1)$ and $f: H \longrightarrow R$ be a proper function. Then,

(1) $f$ is convex if

$$
f(\delta x+(1-\delta) y) \leq \delta f(x)+(1-\delta) f(y), \forall x, y \in H .
$$

(2) $f$ is strongly convex with constant $\sigma$, where $\sigma>0$, if

$$
\begin{aligned}
& f(\delta x+(1-\delta) y)+\frac{\sigma}{2} \delta(1-\delta) x-y^{2} \\
& \leq \delta f(x)+(1-\delta) f(y), \forall x, y \in H .
\end{aligned}
$$

(3) A vector $\omega \in H$ is a subgradient of $f$ at a point $x$ if

$$
f(y) \geq f(x)+\omega, y-x, \forall y \in H .
$$

(4) The set of all subgradients of a convex function $f: H \longrightarrow R$ at $x \in H$, denoted by $\partial f(x)$, is called the subdifferential of $f$ and is defined by

$$
\partial f(x)=\{\omega \in H: f(y) \geq f(x)+\omega, y-x \text {, for each } y \in H\} .
$$

(5) If $\partial f(x) \neq 0, f$ is said to be subdifferentiable at $x$.

If the function $f$ is continuously differentiable, then $\partial f(x)=\{\nabla f(x)\}$. The convex function is subdifferentiable everywhere.

Definition 3. Let $f: H \longrightarrow[-\infty,+\infty)$ be a proper function.

(1) $f$ is lower semicontinuous (lsc) at $x$ if $x^{k} \longrightarrow x$ implies

$$
f(x) \leq \liminf _{k \longrightarrow \infty} f\left(x^{k}\right)
$$

(2) $f$ is weakly lower semicontinuous $(\omega$-lsc) at $x$ if $x^{k} \rightarrow x$ implies

$$
f(x) \leq \liminf _{k \longrightarrow \infty} f\left(x^{k}\right) .
$$


(3) $f$ is lower semicontinuous on $H$ if it is lower semicontinuous at every point $x \in H$ and $f$ is weakly lower semicontinuous on $H$ if it is weakly lower semicontinuous at every point $x \in H$.

Lemma 2 (see [20]). Let $C$ and $Q$ be closed convex subsets of real Hilbert spaces $H_{1}$ and $H_{2}$, respectively, and $f: H_{1} \longrightarrow R$ is given by $f(x)=\left(I-P_{Q}\right) A x^{2}$, where $A: H_{1} \longrightarrow H_{2}$ be a bounded linear operator. Then, for $\delta>0$ and $x^{*} \in H_{1}$, the following statements are equivalent.

(1) The point $x^{*}$ solves the SFP (1), i.e., $x^{*} \in\{x \in C: A x \in Q\}$.

(2) The point $x^{*}$ is the fixed point of the mapping $P_{C}(I-\delta \nabla f)$.

(3) The point $x^{*}$ solves the variational inequality problem with respect to the gradient of $f$, that is, find a point $x^{*} \in C$ such that

$$
\nabla f(x), y-x \geq 0, \forall y \in C .
$$

Lemma 3 (see [6]). Let $H_{1}$ and $H_{2}$ be real Hilbert spaces, and $f: H_{1} \longrightarrow R$ is given by $f(x)=1 / 2\left(I-P_{Q}\right) A x^{2}$, where $Q$ is closed convex subset of $H_{2}$ and $A: H_{1} \longrightarrow H_{2}$ is a bounded linear operator. Then, the following assertions hold:

(1) $f$ is convex and differentiable

(2) $f$ is weakly lower semicontinuous on $H_{1}$

(3) $\nabla f(x)=A^{T}\left(I-P_{Q}\right) A x$, for $x \in H_{1}$

(4) $\nabla f(x) \quad$ is $\quad A^{2}$-Lipschitz,

i.e., $\nabla f(x)-\nabla f(y) \leq A^{2} x-y, \forall x, y \in H_{1}$

Definition 4. Let $\left\{\Lambda_{k}\right\}$ be a real sequence. Then, $\left\{\Lambda_{k}\right\}$ decreases at infinity if there exists $k_{0} \in \mathbb{N}$ such that $\Lambda_{k+1} \leq \Lambda_{k}$, for $k \geq k_{0}$. In other words, the sequence $\left\{\Lambda_{k}\right\}$ does not decrease at infinity if there exists a subsequence $\left\{\Lambda_{k_{t}}\right\}_{t \geq 1}$ of $\left\{\Lambda_{k}\right\}$ such that $\Lambda_{k_{t}}<\Lambda_{k_{t}+1}$, for all $t \geq 1$.

Lemma 4. Let $\left\{\Lambda_{k}\right\}$ be a sequence of real numbers that does not decrease at infinity. Also, consider the sequence of integers $\{\varphi(k)\}_{k \geq k_{0}}$ defined by

$$
\varphi(k)=\max \left\{m \in \mathbb{N}: m \leq k, \Lambda_{m} \leq \Lambda_{m+1}\right\} .
$$

Then, $\{\varphi(k)\}_{k \geq k_{0}}$ is a nondecreasing sequence verifying $\lim _{k \rightarrow \infty} \varphi(k)=\infty$, and for all $k \geq k_{0}$, the following two estimates hold:

$$
\Lambda_{\varphi(k)} \leq \Lambda_{\varphi(k)+1} \text { and } \Lambda_{k} \leq \Lambda_{\varphi(k)+1}
$$

Lemma 5 (see [21]). Let $\left\{s_{k}\right\}$ be a sequence of nonnegative real numbers satisfying the following relation:

$$
s_{k+1} \leq\left(1-\sigma_{k}\right) s_{k}+\sigma_{k} \mu_{k}+\beta_{k}, k \geq 1,
$$

where $\left\{\sigma_{k}\right\},\left\{\mu_{k}\right\}$, and $\left\{\beta_{k}\right\}$ satisfy the conditions:

$$
\begin{aligned}
& \text { (1) }\left\{\sigma_{k}\right\} \subset[0,1], \sum_{k=1}^{\infty} \sigma_{k}=\infty, \\
& \text { (2) } \limsup _{k \longrightarrow \infty} \mu_{k} \leq 0, \\
& \text { (3) } \beta_{k} \geq 0 \sum_{k=1}^{\infty} \beta_{k}<\infty \text { Then, } \underset{k \longrightarrow \infty}{\limsup } s_{k}=0 .
\end{aligned}
$$

\section{Main Results}

In this section, we consider a general case of the SFPwMOS (11), where the nonempty, closed, and convex sets $C$ and $Q_{i}(i=1, \ldots, N)$ are given by level sets of convex functions as in [7]. Throughout this section, we assume that $c: H \longrightarrow \mathbb{R}$ and $q_{i}: H_{i} \longrightarrow \mathbb{R}$ are lower semicontinuous convex functions and the sets $C$ and $Q_{i}$ are given by

$$
C:=\{x \in H: c(x) \leq 0\} \text { and } Q_{i}:\left\{y \in H_{i}: q_{i}(y) \leq 0\right\} .
$$

The relaxed set (half spaces) $C_{k}$ at $x^{k}$ is defined as

$$
C_{k}:=\left\{x \in H: c\left(x^{k}\right) \leq \xi^{k}, x^{k}-x\right\}
$$

where $\xi^{k} \in \partial c\left(x^{k}\right)$.

The relaxed set (half spaces) $Q_{i}^{k}(i=1, \cdots, N)$ at $x^{k}$ are defined as

$$
\begin{gathered}
Q_{i}^{k}:=\left\{x \in H: c\left(x^{k}\right) \leq \xi^{k}, x^{k}-x\right\}, \\
\left\{y \in H_{i}: q_{i}\left(B_{i} x^{k}\right) \leq \eta_{i}^{k}, B_{i} x^{k}-y\right\},
\end{gathered}
$$

where $\eta_{i}^{k} \in \partial q_{i}\left(B_{i} x^{k}\right)$. It follows that $C \subset C_{k}$ and $Q_{i} \subset Q_{i}^{k}$ hold, for every $k \geq 0$. Now, we define the following (relaxed) proximity function; for $x \in H$,

$$
g_{k}(x):=\frac{1}{2} \sum_{i=1}^{N}\left(I-P_{Q_{i}^{k}}\right) B_{i} x^{2} .
$$

We note that $g_{k}($.$) is differentiable with its gradient$ given by

$$
\nabla g_{k}(x):=\sum_{i}^{N} B_{i}^{T}\left(I-P_{Q_{i}^{k}}\right) B_{i} x,
$$

where each $Q_{i}^{k}$ are half spaces given in (37). We note that $g_{k}$ is weakly lower semicontinuous, convex, and differentiable function and $\nabla g_{k}$ is Lipschitz continuous.

Now, we present an inertial self-adaptive relaxed $C Q$ algorithm.

We can see that Algorithm 1 terminates at some iterate (say $k$ ) when $\nabla g_{k}\left(x^{k}\right)=0$.

The following lemma is important for the convergence analysis.

Lemma 6. If $z^{k}:=x^{k}+\theta_{k}\left(x^{k}-x^{k-1}\right)$, where $0 \leq \theta_{k}<1$, for all $k \in \mathbb{N}$, then, for all $z \in H$,

$$
z^{k}-z^{2} \leq x^{k}-z^{2}+\theta_{k}\left(x^{k}-z^{2}-x^{k-1}-z^{2}\right)+2 \theta_{k} x^{k}-x^{k-12} .
$$


Initialization: choose positive sequences $0 \leq \theta_{k}<1,\left\{\rho_{k}\right\} \subset(0,4),\left\{\beta_{k}\right\} \subset(0,1),\left\{\delta_{k}\right\} \subset[0,1)$ and $\left\{\varepsilon_{k}\right\} \subset(0,1)$ such that $\beta_{k}+\delta_{k}+\varepsilon_{k}=1$. Let $u \in H$ be a fixed point. Select arbitrary starting points $x^{0}, x^{1} \in H$, and set $k=0$

Step 1: given the current iterate $x^{k} \in H$, if $\nabla g_{k}\left(x^{k}\right)=0$, for some $k \in \mathbb{N}$, then stop. Otherwise, continue and calculate $\tau_{k}:=\rho_{k} g_{k}\left(z^{k}\right) / \nabla g_{k}\left(z^{k}\right)^{2}$.

Step 2: compute the next iterate as

$$
\left\{\begin{array}{l}
z^{k}=x^{k}+\theta_{k}\left(x^{k}-x^{k-1}\right) \\
x^{k+1}:=P_{C_{k}}\left(\beta_{k} u+\delta_{k} z^{k}+\varepsilon_{k}\left(z^{k}-\tau_{k} \nabla g_{k}\left(z^{k}\right)\right)\right)
\end{array}\right.
$$

where $C_{k}$ is the half space given as in (36)

Algorithm 1: Inertial self-adaptive relaxed CQ algorithm for SFPwMOS.

Proof. Using the identity $2 a, b=a^{2}+b^{2}-a-b^{2}$, we have

$$
\begin{aligned}
z^{k}-z^{2}= & x^{k}-z+\theta_{k}\left(x^{k}-x^{k-1}\right)^{2} \\
= & x^{k}-z^{2}+2 \theta_{k} x^{k}-z, x^{k}-x^{k-1}+\theta_{k}^{2} x^{k}-x^{k-12}, \\
= & x^{k}-z^{2}+\theta_{k} x^{k}-z^{2}+x^{k}-x^{k-12}-x^{k-1} \\
& -z^{2}+\theta_{k}^{2} x^{k}-x^{k-12}, \\
= & x^{k}-z^{2}+\theta_{k}\left(x^{k}-z^{2}-x^{k-1}-z^{2}\right) \\
& +\theta_{k}\left(1+\theta_{k}\right) x^{k}-x^{k-12}, \\
\leq & x^{k}-z^{2}+\theta_{k}\left(x^{k}-z^{2}-x^{k-1}-z^{2}\right) \\
& +2 \theta_{k} x^{k}-x^{k-12} .
\end{aligned}
$$

Next, we show the following strong convergence theorem for Algorithm 1.

Theorem 1. Let $H, H_{i}, i=1, \ldots, N$, be real Hilbert spaces and let $B_{i}: H \longrightarrow H_{i}, i=1, \ldots, N$, be bounded linear operators. Denote $\Gamma$ as the solution set of the problem (11). Let $C$ and $Q_{i}, i=1, \ldots, N$, be nonempty, closed, and convex subsets of $H$ and $H_{i}, i=1, \ldots, N$, respectively. Assume that the SFPwMOS (11) is consistent. Suppose the sequences $\left\{\rho_{k}\right\}$, $\left\{\beta_{k}\right\},\left\{\delta_{k}\right\}$, and $\left\{\varepsilon_{k}\right\}$ in Algorithm 1 satisfy the following conditions:

$$
\begin{aligned}
& \text { (A1) } \lim _{k \rightarrow \infty} \beta_{k}=0 \\
& \text { and } \sum_{k=1}^{\infty} \beta_{k}=+\infty \frac{\beta_{\mathrm{k}+1}}{\beta_{k}} \leq l, l \text { is a constan } t, \\
& \left(\text { A2) } 0<\liminf _{k \longrightarrow \infty} \varepsilon_{k} \leq \limsup _{k \longrightarrow \infty} f \varepsilon_{k}<1,\right. \\
& \left(\text { A3) } \liminf _{k \longrightarrow \infty} \rho_{k}\left(4-\rho_{k}\right)>0,\right. \\
& \left(\text { A4) } \lim _{k \longrightarrow \infty} \frac{\theta_{k}}{\beta_{k}}\left\|x^{k}-x^{k-1}\right\|=0 .\right.
\end{aligned}
$$

Then, the sequence $\left\{x^{k}\right\}$ generated by Algorithm 1 converges strongly to the solution $p \in \Gamma$, where $p=P_{\Gamma}(u)$.

Proof. We may assume that Algorithm 1 does not terminate in a finite number of iterations. Thus, $\nabla g_{k}\left(x^{k}\right) \neq 0$, for all $k \geq 0$. Denote $\Gamma$ as the solution set of problem (11). In the consistent case of problem (11), $\Gamma$ is nonempty, closed, and convex. Thus, the metric projection $P_{\Gamma}$ is well defined.

Let $p \in \Gamma$ and set $s^{k}=z^{k}-\tau_{k} \nabla g_{k}\left(z^{k}\right)$. Note that $I-\mathrm{P}_{Q_{i}^{k}}$, for each $i=1, \ldots, N$, is firmly nonexpansive and $\nabla g_{k}(p)=$ 0 . Hence, we have, from Lemma 1 , that

$$
\begin{aligned}
\left\langle\nabla g_{k}\left(z^{k}\right), z^{k}-p\right\rangle & =\left\langle\sum_{i=1}^{N} B_{i}^{T}\left(I-P_{Q_{i}^{k}}\right) B_{i} z^{k}, z^{k}-p\right\rangle, \\
& =\sum_{i=1}^{N} B_{i}^{T}\left(I-P_{Q_{i}^{k}}\right) B_{i} z^{k}, z^{k}-p, \\
& =\sum_{i=1}^{N}\left(I-P_{Q_{i}^{k}}\right) B_{i} z^{k}, B_{i} z^{k}-B_{i} p, \\
& \geq \sum_{i=1}^{N}\left(I-P_{Q_{i}^{k}}\right) B_{i} z^{k 2}=2 g_{k}\left(z^{k}\right),
\end{aligned}
$$

which implies that

$$
\begin{aligned}
\left\|s^{k}-p\right\|^{2} & =\left\|z^{k}-p-\tau_{k} \nabla g_{k}\left(z^{k}\right)\right\|^{2}, \\
& =z^{k}-p^{2}+\tau_{k}^{2} \nabla g_{k}\left(z^{k}\right)^{2}-2 \tau_{k} \nabla g_{k}\left(z^{k}\right), z^{k}-p \\
& \leq z^{k}-p^{2}+\frac{\rho_{k}^{2} g_{k}^{2}\left(z^{k}\right)}{\nabla g_{k}\left(z^{k}\right)^{2}}-\frac{2 \rho_{k} g_{k}\left(z^{k}\right)}{\nabla g_{k}\left(z^{k}\right)^{2}}\left(2 g_{k}\left(z^{k}\right)\right) \\
& =z^{k}-p^{2}+\frac{\rho_{k}^{2} g_{k}^{2}\left(z^{k}\right)}{\nabla g_{k}\left(z^{k}\right)^{2}}-\frac{4 \rho_{k} g_{k}^{2}\left(z^{k}\right)}{\nabla g_{k}\left(z^{k}\right)^{2}} \\
& =z^{k}-p^{2}-\rho_{k}\left(4-\rho_{k}\right) \frac{g_{k}^{2}\left(z^{k}\right)}{\nabla g_{k}\left(z^{k}\right)^{2}} .
\end{aligned}
$$

Using condition (A3), we have

$$
s^{k}-p^{2} \leq z^{k}-p^{2}, \forall k \geq 0
$$


Next, we show $\left\{z^{k}\right\}$ is bounded. Since $p \in \Gamma \subset C_{k}$ and the projection operator $P_{C_{k}}$ is nonexpansive, we obtain from Algorithm 1 and (48) that

$$
\begin{aligned}
x^{k+1}-p & =P_{C_{k}}\left(\beta_{k} u+\delta_{k} z^{k}+\varepsilon_{k}\left(z^{k}-\tau_{k} \nabla g_{k}\left(z^{k}\right)\right)\right)-p, \\
& \leq \beta_{k} u-p+\delta_{k} z^{k}-p+\varepsilon_{k} s^{k}-p, \\
& \leq \beta_{k} u-p+\delta_{k} z^{k}-p+\varepsilon_{k} z^{k}-p, \\
& =\beta_{k} u-p+\left(1-\beta_{k}\right) z^{k}-p, \\
& =\beta_{k} u-p+\left(1-\beta_{k}\right) x^{k}+\theta_{k}\left(x^{k}-x^{k-1}\right)-p, \\
& \leq \beta_{k} u-p+\left(1-\beta_{k}\right) x^{k}-p+\theta_{k}\left\|x^{k}-x^{k-1}\right\|, \\
& =\left(1-\beta_{k}\right)\left\|x^{k}-p\right\|+\beta_{k}\left[\|u-p\|+\frac{\theta_{k}}{\beta_{k}}\left\|x^{k}-x^{k-1}\right\|\right] .
\end{aligned}
$$

Condition (A4) implies the sequence $\theta_{k} / \beta_{k} x^{k}-x^{k-1}$ is bounded. Let an upper bound of $\left\{u-p+\theta_{k} / \beta_{k} x^{k}-x^{k-1}\right\}$ be M. Then, we rewrite (49) as

$$
x^{k+1}-p \leq \max \left\{x^{k}-p, M\right\},
$$

By induction, we have that

$$
x^{k+1}-p \leq \max \left\{x^{1}-p, M\right\} .
$$

Hence, $\left\{x^{k}-p\right\}$ is bounded, so is $\left\{z^{k}-p\right\}$ and $\left\{s^{k}-p\right\}$. And, $\left\{z^{k}\right\}$ is bounded. Consequently, $\left\{s^{k}\right\}$ and $\left\{B_{i} z^{k}\right\}_{i=1}^{N}$ are also bounded. The rest of the proof will be divided into two parts.

Case 1. Suppose that there exists $k_{0} \in \mathbb{N}$ such that $\left\{x^{k}-p^{2}\right\}_{k=k_{0}}^{\infty}$ is nonincreasing. Then, $\left\{x^{k}-p^{2}\right\}_{k=1}^{\infty}$ converges and $x^{k}-p^{2}-x^{k+1}-p^{2} \longrightarrow 0$ as $k \longrightarrow \infty$. From (47), we obtain

$$
\rho_{k}\left(4-\rho_{k}\right) \frac{\mathrm{g}_{k}^{2}\left(z^{k}\right)}{\nabla \mathrm{g}_{k}\left(z^{k}\right)^{2}} \leq z^{k}-p^{2}-s^{k}-p^{2} .
$$

Since $p \in \Gamma \subset C_{k}$ and the projection operator $P_{C_{k}}$ is nonexpansive, from Algorithm 1, we obtain

$$
\begin{aligned}
x^{k+1}-p^{2} & =P_{C_{k}}\left(\beta_{k} u+\delta_{k} z^{k}+\varepsilon_{k} s_{k}\right)-p^{2}, \\
& \leq\left(\beta_{k} u+\delta_{k} z^{k}+\varepsilon_{k} s_{k}\right)-p^{2}, \\
& \leq \beta_{k} u-p^{2}+\delta_{k} z^{k}-p^{2}+\varepsilon_{k} s_{k}-p^{2} .
\end{aligned}
$$

Since $\beta_{k}+\delta_{k}+\varepsilon_{k}=1$, from (53) and using the inequality $x+y^{2} \leq x^{2}+2 y, x+y$, we have the following estimation:

$$
\begin{aligned}
z^{k}-p^{2}-s^{k}-p^{2} & \leq \frac{\beta_{k}}{\varepsilon_{k}} \mathrm{u}-p^{2}+\frac{1-\beta_{k}}{\varepsilon_{k}} z^{k}-p^{2}-\frac{1}{\varepsilon_{k}} x^{k+1}-p^{2}, \\
& =\frac{\beta_{k}}{\varepsilon_{k}} \mathrm{u}-p^{2}-\frac{\beta_{k}}{\varepsilon_{k}} z^{k}-p^{2}+\frac{1}{\varepsilon_{k}}\left[z^{k}-p^{2}-x^{k+1}-p^{2}\right], \\
& \leq \frac{\beta_{k}}{\varepsilon_{k}} \mathrm{u}-p^{2}+\frac{1}{\varepsilon_{k}}\left[z^{k}-p^{2}-x^{k+1}-p^{2}\right], \\
& =\frac{1}{\varepsilon_{k}}\left[\beta_{k} \mathrm{u}-p^{2}+\left[z^{k}-p^{2}-x^{k+1}-p^{2}\right]\right], \\
& =\frac{1}{\varepsilon_{k}}\left[\beta_{k} \mathrm{u}-p^{2}+\left[x^{k}-p+\theta_{k}\left(x^{k}-x^{k-1}\right)^{2}-x^{k+1}-p^{2}\right]\right], \\
& \leq \frac{1}{\varepsilon_{k}}\left[\beta_{k} \mathrm{u}-p^{2}+\left[x^{k}-p^{2}+2 \theta_{k} x^{k}-x^{k-1}, z^{k}-p-x^{k+1}-p^{2}\right]\right], \\
& \leq \frac{1}{\varepsilon_{k}}\left[\beta_{k} \mathrm{u}-p^{2}+\left[x^{k}-p^{2}+2 \theta_{k} x^{k}-x^{k-1} z^{k}-p-x^{k+1}-p^{2}\right]\right], \\
& =\frac{1}{\varepsilon_{k}}\left[\beta_{k} \mathrm{u}-p^{2}+\left[x^{k}-p^{2}-x^{k+1}-p^{2}+2 \theta_{k} x^{k}-x^{k-1} z^{k}-p\right]\right] .
\end{aligned}
$$

Combining (52) and (54), we obtain 


$$
\begin{aligned}
\rho_{k}(4 & \left.-\rho_{k}\right) \frac{g_{k}^{2}\left(z^{k}\right)}{\nabla g_{k}\left(z^{k}\right)^{2}} \leq z^{k}-p^{2}-s^{k}-p^{2}, \\
\leq & \frac{1}{\varepsilon_{k}}\left[\beta_{k} u-p^{2}+\left[x^{k}-p^{2}-x^{k+1}-p^{2}\right.\right. \\
& \left.\left.+2 \theta_{k} x^{k}-x^{k-1} z^{k}-p\right]\right] .
\end{aligned}
$$

By condition (A4), $\beta_{k} \in(0,1)$ imply the sequence $\lim _{k \longrightarrow \infty} \theta_{k} x^{k}-x^{k-1} \longrightarrow 0$, and we have proved $\left\{z^{k}-p\right\}$ is bounded, so $2 \theta_{k} x^{k}-x^{k-1} z^{k}-p \longrightarrow 0$. By conditions (A2) and (A3) and (55), we have, as $k \longrightarrow \infty$,

$$
\begin{aligned}
0< & \rho_{k}\left(4-\rho_{k}\right) \frac{\mathrm{g}_{k}^{2}\left(z^{k}\right)}{\nabla \mathrm{g}_{k}\left(z^{k}\right)^{2}}, \\
\leq & \frac{1}{\varepsilon_{k}}\left[\beta_{k} \mathrm{u}-p^{2}+\left[x^{k}-p^{2}-x^{k+1}-p^{2}\right.\right. \\
& \left.\left.+2 \theta_{k} x^{k}-x^{k-1} z^{k}-p\right]\right] \longrightarrow 0,
\end{aligned}
$$

which implies that

$$
\lim _{k \rightarrow \infty} \frac{\mathrm{g}_{k}^{2}\left(z^{k}\right)}{\nabla \mathrm{g}_{k}\left(z^{k}\right)^{2}}=0 .
$$

We note that, for each $i=1, \ldots, N, B_{i}^{T}\left(I-P_{Q_{i}^{k}}\right) B_{i}($.$) is$ Lipschitz continuous. Since the sequence $\left\{z^{k}\right\}$ is bounded and

$$
\begin{aligned}
& B_{i}^{T}\left(I-P_{Q_{i}^{k}}\right) B_{i} z^{k}=B_{i}^{T}\left(I-P_{Q_{i}^{k}}\right) B_{i} z^{k} \\
& \quad-B_{i}^{T}\left(I-P_{Q_{i}^{k}}\right) B_{i} p \leq\left(\max _{1 \leq i \leq N} B_{i}\right) z^{k}-p,
\end{aligned}
$$

for all $i=1, \ldots, N$, we have the sequence $\left\{B_{i}^{T}\left(I-P_{Q_{i}^{k}}\right) B_{i} z^{k}\right\}_{i=1}^{\infty}$ is bounded. Hence, $\left\{\nabla g_{k}\left(z^{k}\right)\right\}_{k=1}^{\infty}$ is bounded. Consequently, we have from (57) that

$$
\lim _{k \longrightarrow \infty}\left(I-P_{Q_{i}^{k}}\right) B_{i} z^{k}=0
$$

For

$$
z^{k}-x^{k}=x^{k}+\theta_{k}\left(x^{k}-x^{k-1}\right)-x^{k}=\theta_{k} x^{k}-x^{k-1},
$$

which implies

$$
z^{k}-x^{k} \longrightarrow 0 \text {, as } k \longrightarrow \infty,
$$

for each $i=1, \ldots, N$, since $s^{k}=z^{k}-\tau_{k} \nabla g_{k}\left(z^{k}\right)$, we have from (59) and (61) that

$$
\begin{aligned}
& s^{k}-z^{k}=\tau_{k} \nabla g_{k}\left(z^{k}\right) \longrightarrow 0, \text { as } k \longrightarrow \infty, \\
& s^{k}-x^{k} \leq s^{k}-z^{k}+z^{k}-x^{k} \longrightarrow 0, \text { as } k \longrightarrow \infty .
\end{aligned}
$$

That is,

$$
\begin{array}{r}
\lim _{k \longrightarrow \infty} s^{k}-x^{k}=0, \\
\lim _{k \longrightarrow \infty} B_{i}^{T}\left(I-P_{Q_{i}^{k}}\right) B_{i} z^{k}=0
\end{array}
$$

Let

$$
l^{k}=\beta_{k} u+\delta_{k} z^{k}+\varepsilon_{k} s^{k}=\beta_{k} u+\left(1-\beta_{k}\right) v^{k},
$$

where

$$
v^{k}=\frac{\delta_{k}}{1-\beta_{k}} z^{k}+\frac{\varepsilon_{k}}{1-\beta_{k}} s^{k} .
$$

This gives

$$
\begin{aligned}
& v^{k}-z^{k} \leq \frac{\varepsilon_{k}}{1-\beta_{k}} s^{k}-z^{k} \longrightarrow 0, k \longrightarrow \infty, \\
& \left\|l^{k}-v^{k}\right\| \leq \beta_{k}\left\|u-v^{k}\right\| \longrightarrow 0, k \longrightarrow \infty, \\
& l^{k}-z^{k} \leq l^{k}-v^{k}+v^{k}-z^{k} \longrightarrow 0, k \longrightarrow \infty, \\
& l^{k}-x^{k} \leq l^{k}-z^{k}+z^{k}-x^{k} \longrightarrow 0, \text { as } k \longrightarrow \infty .
\end{aligned}
$$

Hence,

$\lim _{k \longrightarrow \infty} l^{k}-z^{k}=\lim _{k \longrightarrow \infty} l^{k}-x^{k}=\lim _{k \longrightarrow \infty} l^{k}-v^{k}=\lim _{k \longrightarrow \infty} v^{k}-z^{k}=0$.

Since $\left\{x^{k}\right\}$ is bounded, there exists a subsequence $\left\{x^{k_{j}}\right\}$ of $\left\{x^{k}\right\}$ such that $\left\{x^{k_{j}}\right\} \rightarrow p^{*} \in \omega_{\omega}\left(x^{k}\right)$. Next, we need to show $p^{*} \in C$ and $B_{i} p^{*} \in Q_{i}$, for each $i=1, \ldots, N$.

We first show that $x^{k+1}-x^{k} \longrightarrow 0$. For $p \in \Gamma$,

$$
\begin{aligned}
\frac{1}{2} x^{k+1}-p^{2}= & \frac{1}{2} x^{k+1}-x^{k}+x^{k}-p^{2}, \\
= & \frac{1}{2} x^{k}-p^{2}+x^{k+1}-x^{k}, x^{k+1}-p-\frac{1}{2} x^{k+1}-x^{k 2}, \\
= & \frac{1}{2} x^{k}-p^{2}+x^{k+1}-l^{k}+l^{k}-x^{k}, x^{k+1} \\
& -p-\frac{1}{2} x^{k+1}-x^{k 2}, \\
= & \frac{1}{2} x^{k}-p^{2}+x^{k+1}-l^{k}, x^{k+1}-p+l^{k} \\
& -x^{k}, x^{k+1}-p-\frac{1}{2} x^{k+1}-x^{k 2},
\end{aligned}
$$

then

$$
\begin{gathered}
\frac{1}{2} x^{k+1}-x^{k 2} \leq \frac{1}{2} x^{k}-p^{2}-\frac{1}{2} x^{k+1}-p^{2}+x^{k+1}-l^{k}, x^{k+1} \\
-p+l^{k}-x^{k}, x^{k+1}-p,
\end{gathered}
$$

from $x^{k+1}-l^{k}, x^{k+1}-p<0$, 


$$
\frac{1}{2} x^{k+1}-x^{k 2} \leq \frac{1}{2} x^{k}-p^{2}-\frac{1}{2} x^{k+1}-p^{2}+l^{k}-x^{k}, x^{k+1}-p .
$$

Since $\quad x^{k}-p^{2}-x^{k+1}-p^{2} \longrightarrow 0 \quad$ as $\quad k \longrightarrow \infty$, $\lim _{k \rightarrow \infty} l^{k}-x^{k}=0$, we obtain

$$
x^{k+1}-x^{k} \longrightarrow 0 \text {, as } k \longrightarrow \infty \text {. }
$$

Since $x^{k_{j}+1} \in C_{k_{j}}$, we obtain

$$
c\left(x^{k_{j}}\right)+\xi^{k_{j}}, x^{k_{j}+1}-x^{k_{j}} \leq 0 \text {. }
$$

Thus,

$$
c\left(x^{k_{j}}\right) \leq-\xi^{k_{j}}, x^{k_{j}+1}-x^{k_{j}} \leq \xi x^{k_{j}+1}-x^{k_{j}},
$$

where $\xi$ satisfies $\xi^{k} \leq \xi$, for all $k$. By virtue of the continuity of function $c$ and $x^{k_{j}+1}-x^{k_{j}} \longrightarrow 0$, we get that

$$
c\left(p^{*}\right)=\lim _{j \longrightarrow \infty} c\left(x^{k_{j}}\right) \leq 0 .
$$

Therefore, $p^{*} \in C$.

Now, we show that $B_{i} p^{*} \in Q_{i}$; to do this, let $h_{i}^{k}=B_{i} z^{k}-$ $P_{Q_{i}^{k}} B_{i} z^{k} \longrightarrow 0$ and let $\eta_{i}^{k}$ be such that $\eta_{i}^{k} \leq \eta$, for all $k$. Since $B_{i} z^{k_{j}}-h_{i}^{k_{j}}=P_{Q_{i}^{k_{j}}}\left(B_{i} z^{k_{j}}\right) \in Q_{i}^{k_{j}}$, we have

$$
q_{i}\left(B_{i} x^{k_{j}}\right)+\eta_{i}^{k_{j}},\left(B_{i} z^{k_{j}}-h_{i}^{k_{j}}\right)-B_{i} x^{k_{j}} \leq 0 .
$$

Hence,

$$
\begin{aligned}
q_{i}( & \left.B_{i} x^{k_{j}}\right) \leq \eta_{i}^{k_{j}}, B_{i} x^{k_{j}}-B_{i} z^{k_{j}}+\eta_{i}^{k_{j}}, h_{i}^{k_{j}} \leq \eta B_{i} x^{k_{j}} \\
& -x^{k_{j}-1}+\eta h_{i}^{k_{j}} \longrightarrow 0 .
\end{aligned}
$$

By the continuity of $q_{i}$ and $B_{i} x^{k_{j}} \longrightarrow B_{i} x^{*}$, we arrive at the conclusion

$$
q_{i}\left(B_{i} p^{*}\right)=\lim _{j \longrightarrow \infty} q_{i}\left(B_{i} x^{k_{j}}\right) \leq 0,
$$

namely, $B_{i} p^{*} \in Q_{i}$, for all $i=1, \ldots, N$. Hence, $p^{*} \in \Gamma$.

Moreover, for $p=P_{\Gamma} u$, we can see that

$$
\begin{aligned}
\limsup _{k \rightarrow \infty} l^{k}-p, u-p= & \lim _{k \longrightarrow \infty} l^{k_{j}}-p, u-p \leq p^{*} \\
& -p, u-p \leq 0 .
\end{aligned}
$$

By Lemma 1 and (65), we have

$$
\begin{aligned}
& x^{k+1}-p^{2}=P_{C}\left(l^{k}\right)-p^{2} \leq l^{k}-p^{2}=\beta_{k} u+\left(1-\beta_{k}\right) v^{k}-p, l^{k}-p \\
&=\beta_{k} u-p, l^{k}-p+\left(1-\beta_{k}\right) v^{k}-p, l^{k}-p \\
& \leq \beta_{k} u-p, l^{k}-p+\left(1-\beta_{k}\right) v^{k}-p l^{k}-p \\
&=\left(1-\beta_{k}\right)\left\|x^{k}+\theta_{k}\left(x^{k}-x^{k-1}\right)-p\right\|^{2}+\beta_{k}\left\langle u-p, l^{k}-p\right\rangle+\frac{\beta_{k}}{2}\|u-p\|^{2}, \\
& \leq\left(1-\beta_{k}\right)\left[\left\|x^{k}-p\right\|^{2}+2 \theta_{k}\left\langle x^{k}-x^{k-1}, z^{k}-p\right\rangle\right]+\beta_{k}\left\langle u-p, l^{k}-p\right\rangle+\frac{\beta_{k}}{2}\|u-p\|^{2}, \\
& \leq\left(1-\beta_{k}\right)\left[\left\|x^{k}-p\right\|^{2}+2 \theta_{k}\left\|x^{k}-x^{k-1}\right\|\left\|z^{k}-p\right\|\right]+\beta_{k}\left\langle u-p, l^{k}-p\right\rangle+\frac{\beta_{k}}{2}\|u-p\|^{2} . \\
& \quad \phi(k)=\max \left\{t \in \mathbb{N}: t \leq k, \Lambda_{t} \leq \Lambda_{t+1}\right\} .
\end{aligned}
$$

By conditions (A1) and (A4), $\beta_{k} \in(0,1)$ imply the sequence $\lim _{k \longrightarrow \infty} \theta_{k} x^{k}-x^{k-1} \longrightarrow 0, \quad \beta_{k} / 2\|u-p\| \longrightarrow 0$, and using (80) in (81) and applying Lemma 5, we obtain

$$
\lim _{k \rightarrow \infty} x^{k}-p^{2}=0 \text {. }
$$

Therefore, as $k \longrightarrow \infty, x^{k} \longrightarrow p=P_{\Gamma} u$

Case 2. Set $\Lambda_{k}=x^{k}-p^{2}$. Assume that $\left\{\Lambda_{k}\right\}$ is not decreasing at infinity. Let $\phi: \mathbb{N} \longrightarrow \mathbb{N}$ be a mapping for all $k \geq k_{0}$ (for some $k_{0}$ large enough) defined by
By Lemma $4,\{\phi(k)\}_{k=k_{0}}^{\infty}$ is a nondecreasing sequence such that $\phi(k) \longrightarrow \infty$ as $k \longrightarrow \infty$ and

$$
\max \left\{\Lambda_{\phi(k)}, \Lambda_{k}\right\} \leq \Lambda_{\phi(k)+1}, \forall k \geq k_{0} .
$$

After a similar conclusion from (59), it is easy to see that

$$
\lim _{k \longrightarrow \infty}\left(I-P_{Q_{i}^{\phi(k)}}\right) B_{i} z^{\phi(k)}=0 .
$$

By the similar argument as the above in Case 1, we conclude immediately that 


$$
\begin{array}{r}
\lim _{k \longrightarrow \infty} B_{i}^{*}\left(I-P_{Q_{i}^{\phi(k)}}\right) B_{i} z^{\phi(k)}=0, \\
\limsup _{k \longrightarrow \infty} l^{\phi(k)}-p, u-p \leq 0 .
\end{array}
$$

Since $\left\{x^{\phi(k)}\right\}$ is bounded, there exists a subsequence of $\left\{x^{\phi(k)}\right\}$, still denoted by $\left\{x^{\phi(k)}\right\}$ which converges weakly to $p^{*}$. By similar argument as above in Case 1 , we conclude immediately that $p^{*} \in C$ and $B_{i} p^{*} \in Q_{i} \Rightarrow p^{*} \in \Gamma$.

From (57), we have that which implies by Lemma 5

$$
\lim _{k \longrightarrow \infty}\left\|x^{\varphi(k)}-p\right\|^{2}=0 \text { and } \lim _{k \longrightarrow \infty} x^{\phi(k)+1}-p^{2}=0 .
$$

Moreover, for $k \geq k_{0}$, it is easy to see that $\Lambda_{\phi(k)}-\Lambda_{\phi(k)+1} \leq 0$ if $k \neq \phi(k)$ (that is, $\left.k \geq \phi(k)\right)$ because, for $\phi(k)+1 \leq m \leq n, \Lambda_{m}>\Lambda_{m+1}$. As a consequence, we obtain

$$
0 \leq \Lambda_{k} \leq \max \left\{\Lambda_{\phi(k)}, \Lambda_{\phi(k)+1}\right\}=\Lambda_{\phi(k)+1}, \forall k \geq k_{0} .
$$

Therefore, we obtain $\lim \Lambda_{k}=0$, that is, $\left\{x^{k}\right\}$ converges strongly to $p$. This completes the proof.

\section{Some Extensive Results}

For the SFPwMOS (11), when $N=1$, it becomes the SFP (1). Thus, we have the following corollary for solving the SFP (1), which is an immediate consequence of Theorem 1.

Corollary 1. Let $H_{1}$ and $H_{2}$ be two real Hilbert spaces, and let $B: H_{1} \longrightarrow H_{2}$ be bounded linear operator. Let $C$ and $Q$ be nonempty, closed, and convex subsets of $H_{1}$ and $H_{2}$, respectively. Assume that $\Omega=C \cap B^{-1}(Q) \neq 0$. Let $u \in H_{1}$ be a fixed point. For any starting point $x^{0}, x^{1} \in H_{1}$, let $\left\{x^{k}\right\}$ be the sequence generated by

$$
\left\{\begin{array}{l}
z^{k}=x^{k}+\theta_{k}\left(x^{k}-x^{k-1}\right), \\
x^{k+1}:=P_{C_{k}}\left(\beta_{k} u+\delta_{k} z^{k}+\varepsilon_{k}\left(z^{k}-\tau_{k} \nabla g_{k}\left(z^{k}\right)\right)\right),
\end{array}\right.
$$

where $\theta_{k}, C_{k}, \tau_{k}$, and $\nabla f_{k}$ are given as in (8). Suppose the sequences $\left\{\beta_{k}\right\},\left\{\delta_{k}\right\}$, and $\left\{\varepsilon_{k}\right\}$ satisfy the conditions in Theorem 1. Then, the sequence $\left\{x^{k}\right\}$ converges strongly to the solution $p \in \Omega$, where $p=P_{\Omega}(u)$.

When we take $u=x^{0}$ in Algorithm 1, we note also the following results regarding to the SFPwMOS (11).

Corollary 2. Let $H, H_{i}, i=1, \ldots, N$, be real Hilbert spaces and let $B_{i}: H \longrightarrow H_{i}, i=1, \ldots, N$, be bounded linear operators. Let $C$ and $Q_{i}, i=1, \ldots, N$, be nonempty, closed, and convex subsets of $H$ and $H_{i}, i=1, \ldots, N$, respectively. Assume that problem (11) is consistent. For any initial guess $x^{0}, x^{1} \in H$, let $\left\{x^{k}\right\}$ be the sequence generated by where $\theta_{k}, C_{k}, \tau_{k}$, and $\nabla g_{k}$ are given by (36) and (39) and Algorithm 1, respectively. Suppose the sequences $\left\{\beta_{k}\right\},\left\{\delta_{k}\right\}$, and $\left\{\varepsilon_{k}\right\}$ satisfy the conditions in Theorem 1. Then, the sequence $\left\{x^{k}\right\}$ generated by (92) strongly converges to the solution $p=P_{\Omega}\left(x^{0}\right) \in \Gamma$.

When we take $\delta_{k} \equiv 0$ in Algorithm 1, we obtain the following result regarding the SFPwMOS (11).

Corollary 3. Let $H, H_{i}, i=1, \ldots, N$, be real Hilbert spaces, and let $T_{i}: H \longrightarrow H_{i}, i=1, \ldots, N$, be bounded linear operators. Let $C$ and $Q_{i}, i=1, \ldots, N$, be nonempty, closed, and convex subsets of $H$ and $H_{i}, i=1, \ldots, N$, respectively. Assume that problem (11) is consistent. For a fixed point $u \in H$ and any initial guess $x^{0} \in H$, let $\left\{x^{k}\right\}$ be the sequence generated by

$$
\left\{\begin{array}{l}
z^{k}=x^{k}+\theta_{k}\left(x^{k}-x^{k-1}\right), \\
x^{k+1}:=P_{C_{k}}\left(\beta_{k} u+\left(1-\beta_{k}\right)\left(z^{k}-\tau_{k} \nabla g_{k}\left(z^{k}\right)\right)\right),
\end{array}\right.
$$

where $\theta_{k}, C_{k}, \tau_{k}$, and $\nabla g_{k}$ are given by (36) and (39) and Algorithm 1, respectively. Suppose the sequences satisfy conditions (A1). Then, the sequence $\left\{x^{k}\right\}$ generated by (93) strongly converges to the solution $p=P_{\Omega}(u) \in \Gamma$.

Of course, when we take $u=x^{0}$, we get the following result regarding the SFPwMOS (11).

Corollary 4. Let $H, H_{i}, i=1, \ldots, N$, be real Hilbert spaces and let $B_{i}: H \longrightarrow H_{i}, i=1, \ldots, N$, be bounded linear operators. Let $C$ and $Q_{i}, i=1, \ldots, N$, be nonempty, closed, and convex subsets of $H$ and $H_{i}, i=1, \ldots, N$, respectively. Assume that problem (11) is consistent. For any initial guess $x^{0} \in H$, let $\left\{x^{k}\right\}$ be the sequence generated by

$$
\left\{\begin{array}{l}
z^{k}=x^{k}+\theta_{k}\left(x^{k}-x^{k-1}\right) \\
x^{k+1}:=P_{C_{k}}\left(\beta_{k} x^{0}+(1-\text { tarts - with (., hskip')] })\right. \text { ")hskip! "substring - }
\end{array}\right.
$$


TABLe 1: Algorithm 1 for different choices of $\theta_{k}$, $\varepsilon$, and $\rho_{k}$.

\begin{tabular}{|c|c|c|c|c|c|}
\hline \multirow{2}{*}{$\varepsilon$} & \multirow{2}{*}{$\theta_{k}$} & \multicolumn{2}{|c|}{$\rho_{k}=3.98$} & \multicolumn{2}{|c|}{$\rho_{k}=3.00$} \\
\hline & & Iter & $E_{k}$ & Iter & $E_{k}$ \\
\hline \multirow{3}{*}{$10^{-3}$} & $\theta_{k}=0.8$ & 5 & $7.0906 e-05$ & \multirow{3}{*}{6} & \multirow{3}{*}{$5.8048 e-05$} \\
\hline & $\theta_{k}=0.3$ & 7 & $6.9397 e-04$ & & \\
\hline & $\theta_{k}=0$ & 9 & $7.5095 e-04$ & & \\
\hline \multirow{3}{*}{$10^{-4}$} & $\theta_{k}=0.8$ & 5 & $7.0906 e-05$ & \multirow{3}{*}{6} & \multirow{3}{*}{$5.8048 e-05$} \\
\hline & $\theta_{k}=0.3$ & 11 & $9.1282 e-05$ & & \\
\hline & $\theta_{k}=0$ & 15 & $9.6150 e-05$ & & \\
\hline \multirow{3}{*}{$10^{-5}$} & $\theta_{k}=0.8$ & 12 & $4.4752 e-06$ & \multirow{3}{*}{14} & \multirow{3}{*}{$2.5296 e-06$} \\
\hline & $\theta_{k}=0.3$ & 16 & $9.1012 e-06$ & & \\
\hline & $\theta_{k}=0$ & 24 & $8.8064 e-06$ & & \\
\hline
\end{tabular}

where $\theta_{k}, C_{k}, \tau_{k}$, and $\nabla g_{k}$ are given by (36) and (39) and Algorithm 1, respectively. Suppose the sequence $\left\{\beta_{k}\right\}$ satisfies conditions (A1). Then, the sequence $\left\{x^{k}\right\}$ generated by (94) strongly converges to the solution $p=P_{\Omega}(u) \in \Gamma$.

Remark 1. In Corollary 4, for the particular case, where $N=1$, the iterative scheme (94) reduced exactly to iterative scheme proposed by $\mathrm{He}$ et al. in Theorem 3.2 of [10].

\section{Numerical Experiment}

In this section, we provide one numerical experiment to illustrate the implementation and efficiency of our proposed method. First, we study the behavior of Algorithm 1 for different choices of $\theta_{k}$ and $\rho_{k}$. Next, we compare Algorithm 1 $\left(\theta_{k}=0.8\right)$ with no inertial Algorithm $1\left(\theta_{k}=0\right)$ and Scheme (17) for different initial points. The numerical results are completed on a standard TOSHIBA laptop with Intel(R) Core(TM) i5-2450M CPU@2.5GHz 2.5 GHz with memory $4 \mathrm{~GB}$. The code is implemented in MATLAB R2020a.

Let

$$
\begin{aligned}
A_{1} & =\left[\begin{array}{ccc}
-0.4 & -0.2 & -0.2 \\
0.4 & 0.5 & -0.1 \\
0.2 & -0.5 & 0.3
\end{array}\right], \\
A_{2} & =\left[\begin{array}{ccc}
0.3 & -0.1 & 0.3 \\
-0.3 & 0.2 & -0.2 \\
0 & 0.2 & 0.3
\end{array}\right], \\
C & =\left\{x \in R^{3} \mid x_{1}-x_{2}^{2}+2 x_{3} \leq 0\right\}, \\
Q_{1} & =\left\{x \in R^{3} \mid x_{1}^{2}+x_{2}-x_{3} \leq 0\right\}, \\
Q_{2} & =\left\{x \in R^{3} \mid x_{1}+x_{2}^{2}-x_{3} \leq 0\right\} .
\end{aligned}
$$

Find $x^{*}$ such that

$$
x^{*} \in \Gamma:=C \cap\left(\cap{ }_{i=1}^{2} A_{i}^{-1}\left(Q_{i}\right)\right) \neq 0 .
$$

Define the error function as

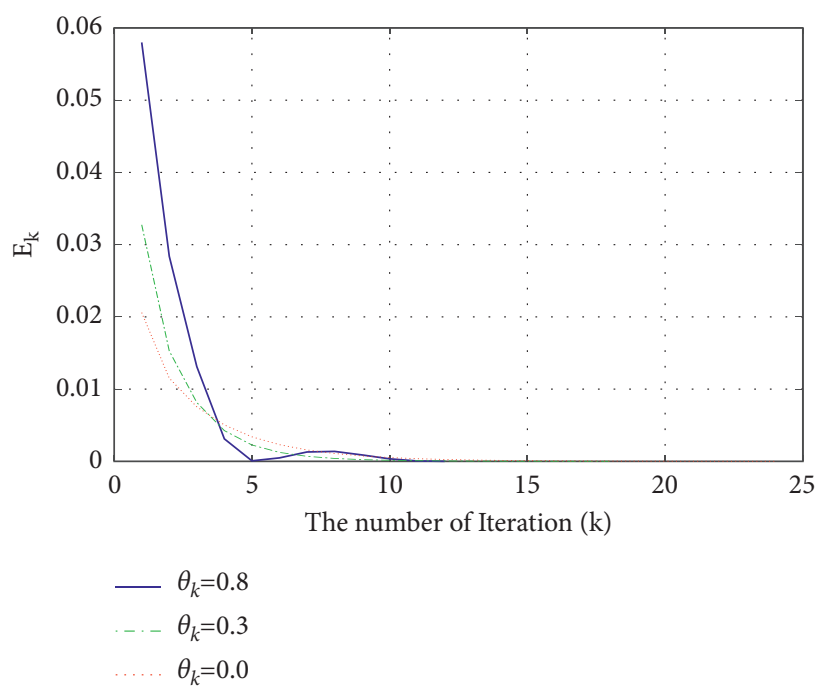

FIgURE 1: Error against iterations for Algorithm 1 with difference choices of $\theta_{k}$, under $\rho_{k}=3.98$ and $\varepsilon=10^{-5}$.

$$
\begin{aligned}
E_{k}= & \frac{1}{3} x^{k}-P_{C_{k}}\left(x^{k}\right)^{2}+\frac{1}{3} A_{1} x^{k}-P_{Q_{1, k}}\left(A_{1} x^{k}\right)^{2} \\
& +\frac{1}{3} A_{2} x^{k}-P_{Q_{2, k}}\left(A_{2} x^{k}\right)^{2} .
\end{aligned}
$$

Note that if $E_{k}=0$ at the $k$ th step, then $x^{k} \in \Gamma$.

For Algorithm 1, for different choices of $\rho_{k}$ and $\theta_{k}$, we choose $\quad \beta_{k}=1 / 9 k+1, \quad \delta_{k}=k / 9 k+1, \quad \varepsilon_{k}=8 k / 9 k+1$, $u=(0.10 .10 .1)^{T}, \quad$ and $\quad x^{0}=(-0.1-0.1-0.1)^{T} \quad$ and $x^{1}=[0.30 .5-0.6]^{T}$. Using $E_{k}<\varepsilon$ as stopping criteria, where $\varepsilon$ is a small enough positive number, the results of the numerical experiment are reported in Table 1. The numerical results can be seen from Table 1 and Figures 1 and 2. In Table 1, "Iter" denotes the number of iterations.

The behavior of the function $E_{k}$ in Table 1 is described in Figures 1 and 2 .

From Table 1 and Figures 1 and 2, we can observe that Algorithm 1 for inertial effect case has less number of iterations than Algorithm 1 for $\theta_{k}=0$ (that is, no inertial effect) case; Algorithm 1 with bigger $\theta_{k}$ has less number of iterations than Algorithm 1 for small case; for the same $\theta_{k}$, big $\rho_{k}$ case has less number of iterations than Algorithm 1 for small case. 


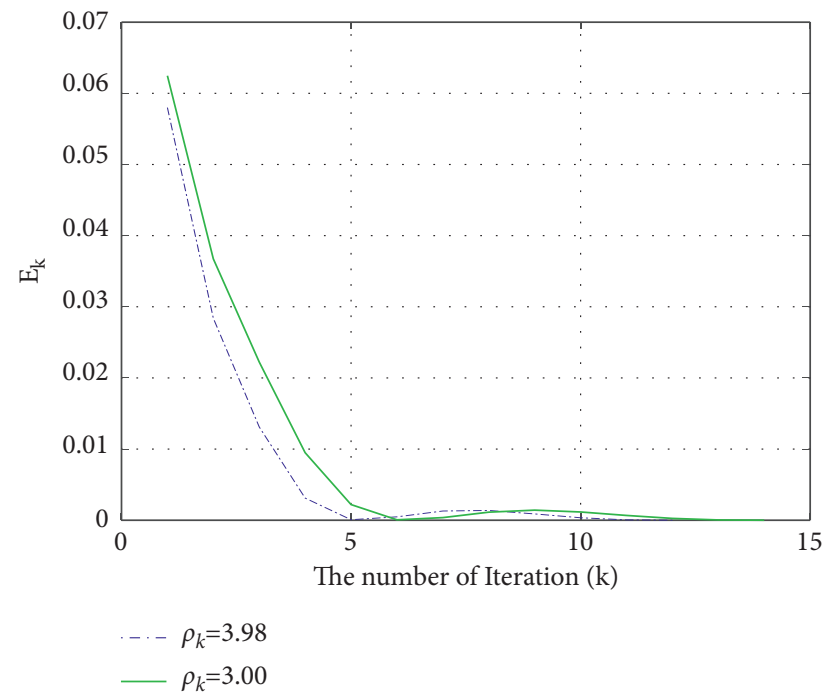

FIgURE 2: Error against iterations for Algorithm 1 with difference choices of $\rho_{k}$, under $\theta_{k}=0.8$ and $\varepsilon=10^{-5}$.

TABle 2: Comparsion of Algorithm 1 and Scheme (97) for different choice initial point for $\varepsilon=10^{-4}$

\begin{tabular}{|c|c|c|c|c|c|c|}
\hline \multirow{2}{*}{ Initiative point $x^{1}$} & \multicolumn{2}{|c|}{ Algorithm 1 with $\theta_{k}=0.8$} & \multicolumn{2}{|c|}{ Algorithm 1 with $\theta_{k}=0.0$} & \multicolumn{2}{|c|}{ (98) } \\
\hline & Iter & $E_{k}$ & Iter & $E_{k}$ & Iter & $E_{k}$ \\
\hline$\left[\begin{array}{lll}0.4 & 0.9-0.9\end{array}\right]$ & 11 & $4.1830 e-06$ & 25 & $9.6999 e-06$ & 51 & $8.8226 e-06$ \\
\hline$\left[\begin{array}{lll}0.9 & 0.3-0.5\end{array}\right]$ & 6 & $1.4290 e-07$ & 22 & $8.6399 e-06$ & 26 & $9.6719 e-06$ \\
\hline$\left[\begin{array}{lll}-0.7 & 0.3-0.2\end{array}\right]$ & 12 & $7.8838 e-06$ & 24 & $9.2013 e-06$ & 48 & $8.4198 e-06$ \\
\hline$\left[\begin{array}{ll}0.1 & 0.1-0.9\end{array}\right]^{\prime}$ & 12 & $1.5353 e-07$ & 26 & $8.7544 e-06$ & 52 & $9.9722 e-06$ \\
\hline
\end{tabular}

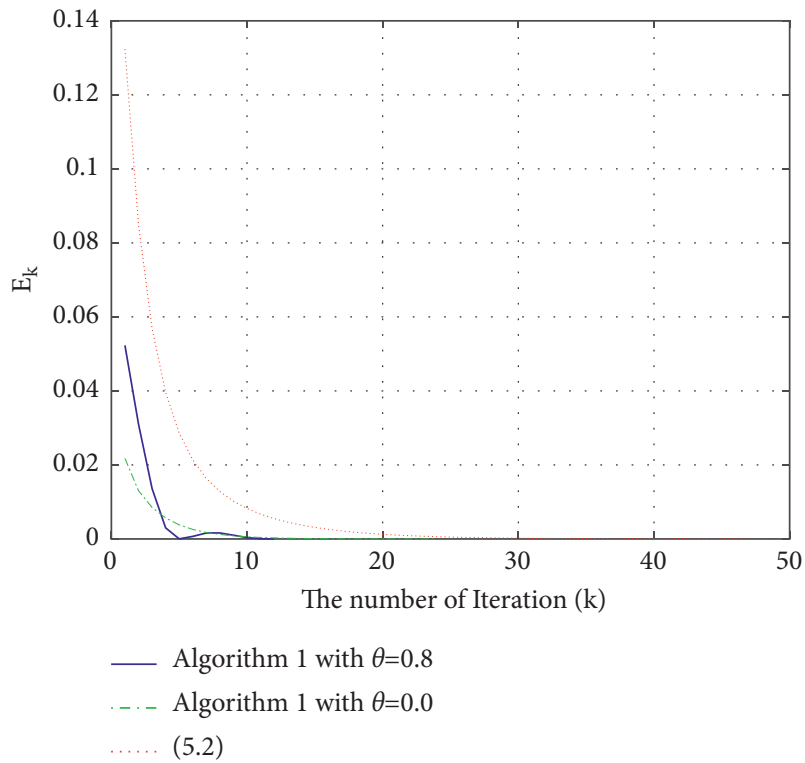

FIGURE 3: Error against of iterations for the comparision of Algorithm 1 and Scheme (97).

Following, we compare our algorithm with iteration (16). For the example, iteration (16) can be written as

$$
\begin{aligned}
x^{k+1}:= & \left.\alpha_{k} f\left(x^{k}\right)+\left(1-\alpha_{k}\right)\right) P_{C}\left(x^{k}-\lambda_{k}\left(B_{1}^{*}\left(I-P_{\mathrm{Q}_{1}}\right)\right) B_{1} x^{k}\right. \\
& \left.+\left(B_{2}^{*}\left(I-P_{\mathrm{Q}^{2}}\right)\right) B_{2} x^{k}\right) .
\end{aligned}
$$

In the process, we take $\alpha_{k}=0.8$ and $f\left(x^{k}\right)=$ $u=(111)^{T} \lambda_{k}=0.00005$ for Scheme (97). We choose $\beta_{k}=$ $1 / 2 k+1, \delta_{k}=\varepsilon_{k}=k / 2 k+1$, and $\rho_{\mathrm{k}}=3$ for Algorithm 1 .

From Table 2 and Figure 3, we can observe that Algorithm 1 has less number of iterations than Scheme (97). Moreover, our iterative method is advantageous over Scheme (98) because Algorithm 1 does not require computing of the operator norm.

\section{Data Availability}

No data were used to support this study.

\section{Conflicts of Interest}

The authors declare that they have no conflicts of interest.

\section{Authors' Contributions}

This entire work has been completed by the authors: Huijuan Jia, Shufen Liu, and Yazheng Dang. The authors read and approved the finial manuscript. 


\section{Acknowledgments}

This research was supported by the National Natural Science Foundation of China (Grant no. 61872126), Henan Province Key Science and Technology Project (Grant no. 192102210123), and Young Backbone Teachers in Universities of Henan Province (Grant no. 2019GGJS061).

\section{References}

[1] Y. Censor and T. Elfving, "A multiprojection algorithm using bregman projections in a product space," Numerical Algorithms, vol. 8, no. 2, pp. 221-239, 1994.

[2] C. Byrne, "Iterative oblique projection onto convex sets and the split feasibility problem," Inverse Problems, vol. 18, no. 2, pp. 441-453, 2002.

[3] G. T. Herman, "Image reconstruction from projections the fundamentals of computerized tomography," 1980.

[4] Y. Censor and A. Segal, "Iterative projection methods in biomedical inverse problems," 2008.

[5] Y. Censor, T. Bortfeld, B. Martin, and A. Trofimov, "A unified approach for inversion problems in intensity-modulated radiation therapy," Physics in Medicine and Biology, vol. 51, no. 10, pp. 2353-2365, 2006.

[6] C. Yair and Tommy, "The multiple-sets split feasibility problem and its applications for inverse problems," Inverse Problems, vol. 21, no. 6, pp. 2071-2084, 2005.

[7] Q. Yang, "The relaxed CQ algorithm solving the split feasibility problem," Inverse Problems, vol. 20, no. 4, pp. 1261-1266, 2004.

[8] G. Lopez, V. Martın-Marquez, and F. H. Wang, "Solving the split feasibility problem without prior knowledge of matrix norms," Inverse Problems, vol. 28, no. 8, pp. 374-389, 2012.

[9] J. Deepho and P Kumam, “A modified halpern's iterative scheme for solving split feasibility problems," Abstract and Applied Analysis, vol. 2012, Article ID 876069, 8 pages, 2012.

[10] Y. Dang and Y. Gao, "The strong convergence of a KM-CQlike algorithm for a split feasibility problem," Inverse Problems, vol. 27, no. 1, Article ID 015007, 2011.

[11] S. N. He and Z. Y. Zhao, "Strong convergence of a relaxed CQ algorithm for the split feasibility problem," Journal of Inequalities and Applications, vol. 2013, no. 1, 11 pages, 2013.

[12] Y. H. Yao and Mihai, Strong Convergence of a Self-Adaptive Method for the Split Feasibility Problem Fixed Point Theory o Applications, Springer, Berlin, Germany, 2013.

[13] S. Reich, M. T. Truong, and T. Mai, "The split feasibility problem with multiple output sets in Hilbert spaces," Optimization Letters, vol. 36, 2020.

[14] S. Suantai, N. Pholasa, N. Pholasa, and P. Cholamjiak, "The modified inertial relaxed CQ algorithm for solving the split feasibility problems," Journal of Industrial and Management Optimization, vol. 14, no. 4, pp. 1595-1615, 2018.

[15] W. Cholamjiak, P. Cholamjiak, and S. Suantai, "An inertial forward-backward splitting method for solving inclusion problems in Hilbert spaces," Journal of Fixed Point Theory and Applications, vol. 20, no. 1, p. 42, 2018.

[16] Q. L. Dong, Y. J. Cho, and L. L. Zhong, "Inertial projection and contraction algorithms for variational inequalities," Journal of Global Optimization, vol. 70, no. 3, pp. 1-18, 2018.

[17] D. R. Sahu, Y. J. Cho, and Q. L. Dong, "Inertial relaxed CQ algorithms for solving a split feasibility problem in Hilbert spaces," Numerical Algorithms, vol. 3, 2020.

[18] Y. Shehu and A. Gibali, "Inertial krasnoselskii-mann method in banach spaces," Mathematics, vol. 8, no. 4, 2020.
[19] Y. Shehu, P. T. Vuong, and P. Cholamjiak, "A self-adaptive projection method with an inertial technique for split feasibility problems in Banach spaces with applications to image restoration problems," Journal of Fixed Point Theory and Applications, vol. 21, no. 2, 2019.

[20] H.-K. Xu, "Iterative methods for the split feasibility problem in infinite-dimensional Hilbert spaces," Inverse Problems, vol. 26, no. 10, Article ID 105018, 2010.

[21] R. I. Bot and E. R. Csetnek, "An inertial Tseng's type proximal algorithm for nonsmooth and nonconvex optimization problems," Journal of Optimization Theory and Applications, vol. 171, no. 2, pp. 600-616, 2016. 